\title{
Context-driven Smart Authoring of Multimedia Content with XSMART
}

\author{
Ansgar Scherp \\ Oldenburg Research and Development Institute \\ for Computer Science Tools and Systems \\ (OFFIS), Oldenburg, Germany \\ ansgar.scherp@offis.de
}

\author{
Susanne Boll \\ University of Oldenburg \\ Department of Computing Science, \\ Oldenburg, Germany \\ boll@informatik.uni-oldenburg.de
}

\begin{abstract}
In recent years, many highly sophisticated multimedia authoring tools have been developed. Up to today, these system's integration of the targeted user context, however, is limited. With our Context-aware Smart Multimedia Authoring Tool (xSMART) we developed a semi-automatic authoring tool that integrates the targeted user context into the different authoring steps and exploits this context to guide the author through the content authoring process. The design of xSMART allows that it can be extended and customized to the requirements of a specific domain by domain-specific wizards. These wizards realize the user interface that meets best the domain-specific requirements and effectively supports the domain experts in creating their content targeted at a specific user context.
\end{abstract}

Categories and Subject Descriptors: H.5.1 [Information Interfaces and Presentation]: Multimedia Information Systems; H.5.4 [Information Interfaces and Presentation]: Hypertext/Hypermedia

General Terms: Design, Human Factors, Experimentation

Keywords: smart multimedia authoring, context

\section{INTRODUCTION}

Multimedia content authoring can be seen as the process in which synchronized multimedia presentations composed of different discrete and continuous media elements are created. The authoring of multimedia content is a challenging task and typically needs an high expertise in both the domain and often also in using the authoring tool, too. Authoring becomes even more challenging when the targeted multimedia presentation needs to meet a specific user context. Then, only the relevant media elements, the proper media formats, and the right modality needs to be chosen such that the end user finally receives a presentation that meets his or her personal interest, the technical infrastructure, and (mobile) end device.

Looking at the field of multimedia authoring today, we find a number of tools ranging from domain expert tools to general purpose authoring tools: Domain expert tools hide as much as possible the technical details of content authoring and are often organized in a wizard-like fashion that

Copyright is held by the author/owner. MM'05, November 6-11, 2005, Singapore. ACM 1-59593-044-2/05/0011. guide the expert in the field through the authoring process. For example the Cardio-OP-Wizard that authors a multimedia book on cardiac surgery [2]. On the other end of the spectrum, we find highly generalized multimedia authoring tools such as Director and Authorware [3]. These domainindependent tools let the authors create very sophisticated multimedia presentations, however, typically require high expertise from them (including programming skills). In the field of adaptive multimedia models there exist authoring tools such as the GR $i$ NS editor [4] for SMIL documents. These tools are still tedious to handle and not practical for the domain experts, and following Bulterman in [1]: "Unfortunately, we have not seen the hoped-for uptake of authoring systems for SMIL or for any other format."

So, multimedia authoring is still an open issue and has become even more challenging with the increase of different devices, platforms, and multimedia formats and at the same time the high demand for personalization of content for the individual user or user group. To contribute to a lesssumptuous authoring process that is aware of the targeted user context, we developed a prototype of a Context-aware Smart Multimedia Authoring Tool (xSMART).

This wizard-based tool integrates context into all steps of the authoring process from the content selection, content composition to the creation of the multimedia presentations in the final presentation formats. However, we did not build yet another authoring tool but designed it such that it can be used and extended by arbitrary domain-specific wizards. xSMART itself is based on our MM4U component framework for creating personalized multimedia content $[5,6]$. In the following, we present the general content driven authoring process, before we come to the design and implementation of our Context-aware Smart Multimedia Authoring Tool and a concrete personalized photo album authoring wizard.

\section{CONTEXT-DRIVEN AUTHORING}

Fig. 1 depicts the general context-driven authoring process of multimedia content and the integration of user context: In the selection phase, the targeted user context can be used to narrow down the different options for the multimedia composition. Hence, the authoring environment presents only those media elements for an inclusion into the presentation that are technically and/or semantically relevant for the presentation. For the composition, the context plays the role of determining the style and layout of the presentation. For transforming the assembled multimedia content to concrete presentation formats only those output formats are offered, that are reasonable for the selected (mobile) end de- 
vice. Hence, the context stands by to relieve the author from decisions and tasks during the authoring process which can be "foreseen" by the targeted user context and, hence, are hidden from the author. What type of context information is actually needed and exploited to support the authoring process depends on the concrete application domain. It can be, e. g., the targeted end device or multimedia player, the user's preferred presentation style and language, as well as any other domain-specific context information.

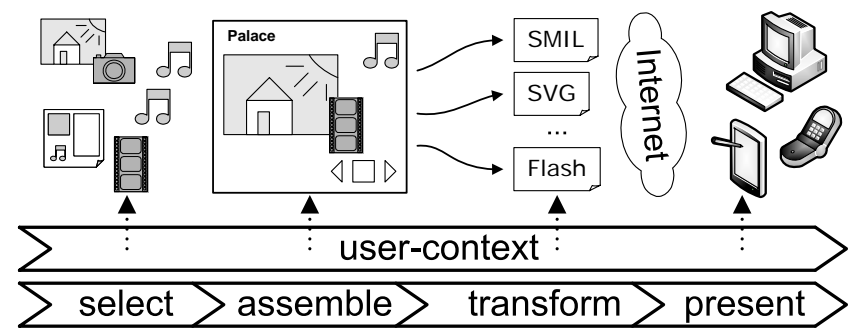

Figure 1: Context-driven multimedia authoring

\section{XSMART ARCHITECTURE}

The xSMART architecture provides an abstraction of general purpose authoring tools and offers only the very basic set of multimedia composition functionality. This includes the selection of media elements and links and the creation of simple layout in a page-oriented fashion. So far the system is not different to existing approaches in the field. However, the design differentiates a concrete authoring wizard from basic authoring features, which again abstract from the concrete composition of a multimedia document.

Fig. 2 illustrates these different levels of multimedia authoring: The basis of this architecture forms our MM4U component framework for creating personalized multimedia content $[5,6]$. On top of it, the xSMART authoring tool adds another level of abstraction with suitable interfaces, targeted at a simplified multimedia authoring. Using the xSMART authoring tool, domain-specific wizards can be developed to support domain experts in their content creation task as presented in Fig.1. These domain-specific wizards guide the domain expert step-by-step through the context-driven authoring process. In contrast of earlier usage of our MM4U framework for the automatic generation of personalized multimedia content, our xSMART authoring tool provides support for the development of context-driven semi-automatic multimedia authoring wizards.

\section{XSMART AUTHORING}

The context-driven authoring wizard supports the author in pre-selecting media elements for the considered domain and according to the profile information of the targeted user or user group. For example with an wizard for creating personalized photo albums, an author could define that only pictures taken during summer time are to be selected and only those, with a good exposure as well as sharpness. Therefore, the xSMART authoring tool creates queries for the underlying profile and media databases of the MM4U framework and passes the results back to the domain-specific wizard. With the photo album wizard, the author can also determine the targeted (mobile) end device. Here, xSMART provides for automatically switching to the best suitable presentation dimension. For the composition of media elements, xSMART defines some useful abstractions of the fine-granular multimedia composition functionalities the MM4U framework provides. These abstractions allow for an as simple as possible composition of media elements and links in time and space in a page-oriented multimedia presentation. For exporting the authored multimedia content, the xSMART tool provides the wizard with a list of concrete presentation formats that are supported by the underlying $\mathrm{MM} 4 \mathrm{U}$ framework and which are supported by the selected end device. Once selected an possible format, the multimedia content is exported and the transformation result can be previewed in the subsequent step. When finishing with the preview of the multimedia presentation, the author can modify or deploy the authored multimedia content.

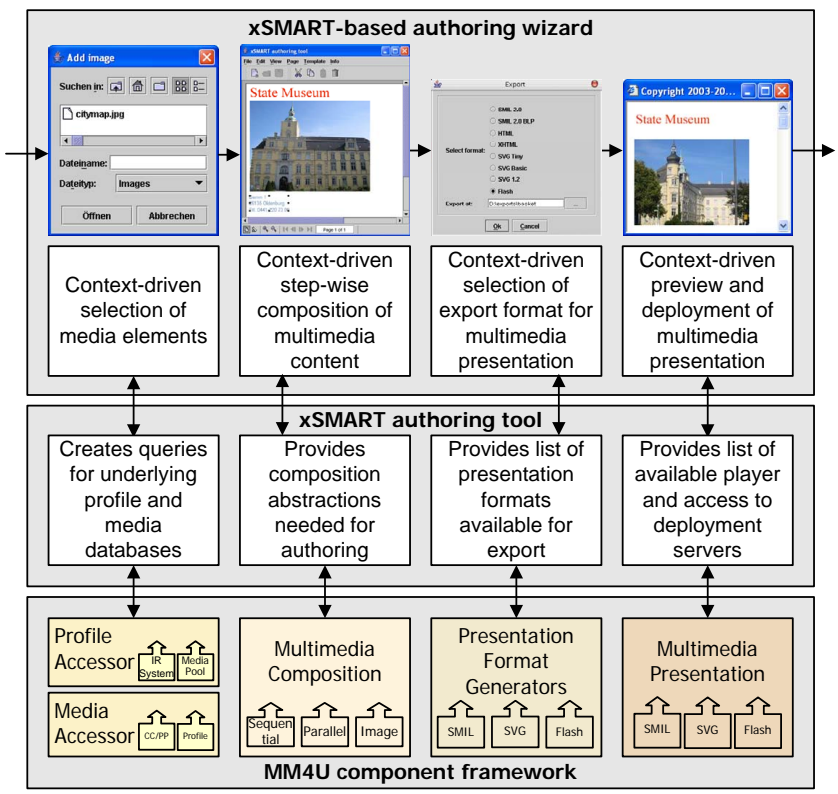

Figure 2: Architecture of the Smart Authoring

\section{CONCLUSIONS}

The presented Context-aware Smart Multimedia Authoring Tool can be seen as first step towards a sophisticated authoring suite of context-driven multimedia content authoring. With xSMART and the underlying MM4U framework, we contribute to a more efficient and faster development of domain-specific tools for multimedia authoring.

Acknowledgements: We kindly thank our students that have been developing the prototype Marko Heuer and Holger Cremer.

\section{REFERENCES}

[1] Bulterman, Hardman: Structured multimedia authoring. ACM TOMCCAP, Vol. 1, No. 1, 2005.

[2] Greiner, Rose: A Web Based Training System for Cardiac Surgery: The Role of Knowledge Management for Interlinking Information Items. In: Proc. of MEDNET 1998, London, UK.

[3] Macromedia, USA. http://www.macromedia.com, 2005.

[4] Oratrix, Netherlands. http://www.oratrix.com, 2005.

[5] Scherp, Boll: Paving the Last Mile for Multi-Channel Multimedia Presentation Generation. In: Procedings of the 11th MMM Conf. Chen, Y.-P. P. (ed.). Melbourne, Australia: IEEE Computer Society, Jan. 2005.

[6] Scherp, Boll: MM4U - A framework for creating personalized multimedia content. In: Managing Multimedia Semantics. Srinivasan, U. \& Nepal, S. (ed.). IRM Press, 2005. 